\title{
The VAD Scheme versus Thalidomide plus VAD for Reduction of Vascular Endothelial Growth Factor in Multiple Myeloma: A Meta-Analysis
}

\author{
Gan-Lin He $\mathbb{D}^{1},{ }^{1}$ Duo-Rong Xu, ${ }^{1}$ Wai-Yi Zou, ${ }^{1}$ Sui-Zhi He, ${ }^{2}$ and Juan Li ${ }^{1}{ }^{1}$ \\ ${ }^{1}$ Department of Hematology, The First Affiliated Hospital, Sun Yat-sen University, Guangzhou, China \\ ${ }^{2}$ Teaching and Research Section of Advanced Mathematics, Xinhua College of Sun Yat-sen University, Guangzhou, China \\ Correspondence should be addressed to Juan Li; 13719209240@163.com
}

Received 8 April 2018; Accepted 2 August 2018; Published 11 November 2018

Academic Editor: Francesco Di Raimondo

Copyright ( $\odot 2018$ Gan-Lin He et al. This is an open access article distributed under the Creative Commons Attribution License, which permits unrestricted use, distribution, and reproduction in any medium, provided the original work is properly cited.

\begin{abstract}
The VAD (vincristine-doxorubicin-dexamethasone) regimen has been used for decades to treat multiple myeloma (MM). Based on reports that vascular endothelial growth factor- (VEGF-) mediated angiogenesis is critical for MM pathogenesis, the antiangiogenic compound thalidomide has been added to VAD (T-VAD). However, it remains unclear whether T-VAD is more efficacious than VAD for serum VEGF reduction or if the difference influences clinical outcome. Pubmed, Cochrane library, China Biomedical Literature (CBM) database, China National Knowledge Infrastructure (CNKI) database, Vip database, and Wanfang database were searched for relevant studies published up to June 2017. RevMan5.2 was used for methodological quality evaluation and data extraction. Thirteen trials (five randomized, seven nonrandomized, and one historically controlled) involving 815 cases were included. Serum VEGF was significantly higher in MM cases than non-MM controls ( $\mathrm{MD}=353.01$, [95\%CI 187.52-518.51], $\mathrm{P}<0.01$ ), and the overall efficacy of T-VAD was higher than that of VAD $(\mathrm{RR}=1.36,[1.21-1.53], \mathrm{P}<0.01)$. Further, T-VAD reduced VEGF to a greater extent than VAD does ( $[\mathrm{MD}=-49.85,[-66.28--33.42], \mathrm{P}<0.01)$. The T-VAD regimen also reduced VEGF to a greater extent in newly diagnosed MM patients than it did in recurrent patients ([MD=-120.20, [-164.60--39.80], $\mathrm{P}<0.01$ ). There was no significant difference in VEGF between T-VAD patients (2 courses) and nontumor controls ( $\mathrm{MD}=175.94,[-26.08-377.95], \mathrm{P}=0.09$ ). Greater serum VEGF reduction may be responsible for the superior efficacy of T-VAD compared to VAD.
\end{abstract}

\section{Introduction}

Multiple myeloma (MM) is a heterogeneous disease characterized by the clonal proliferation of immunoglobulinproducing plasma cells. It accounts for $1 \%$ of all cancers and $10 \%$ of all hematologic malignancies [1-4]. About 86000 new cases of myeloma are diagnosed globally each year $[5,6]$.

Since the 1980s, the VAD (vincristine-doxorubicindexamethasone) regimen has been the preferred therapeutic schedule for MM, with curative efficacy ranging from 50\% to $70 \%$ [7]. Compared to the older MP (melphalan + prednisone) regimen, VAD is faster acting and does not influence the mobilization of stem cells, which is beneficial for subsequent autologous stem cell transplantation and other treatments. An additional advantage of VAD is relatively low cost, so it is widely used for MM treatment in China and other developing countries $[8,9]$.
Vacca et al. [10] first reported a substantial increase in microvessel density (MVD) among MM patients that was strongly correlated with the proliferation of myeloma cells. Subsequent studies revealed that serum vascular endothelial growth factor (VEGF) is elevated in MM patients and overexpressed in MM cell lines [11-13]. Further, low serum VEGF level is correlated with the curative effect of therapy, while high serum VEGF is a major prognostic factor for poor outcome in MM patients [14]. The occurrence, development, invasion, and metastasis of cancer rely on the generation of new tumor vascular networks [15], strongly suggesting that serum VEGF influences the development and prognosis of MM through bone marrow angiogenesis. Therefore, antiangiogenic agents, especially drugs acting against VEGF, have become a major focus in the development of new MM treatments. A large number of studies show that thalidomide 
(THD) can inhibit angiogenesis, suppress cytokine signaling, promote myeloma cell apoptosis, and alter the bone marrow microenvironment for sustained stem cell mobilization [16].

There are many reports on the addition of antiangiogenic drugs (including thalidomide) to VAD chemotherapy for MM treatment. Such studies have shown that this T-VAD regimen decreases VEGF and is an effective curative treatment $[8,17-19]$. However, no large-scale systematic study has compared the T-VAD and VAD regimens for effects on serum VEGF or assessed the relationship between serum VEGF reduction and clinical outcome. Therefore, we conducted a meta-analysis of studies reporting serum VEGF changes in patients treated by VAD and (or) T-VAD and evaluated the association of serum VEGF changes with clinical outcome. The primary objective of this study is to analyze the evidence for VEGF reduction as a mechanism for T-VAD efficacy based on randomized, nonrandomized, and historical controlled trials.

\section{Methods}

2.1. Search Strategy and Selection Criteria. The trials analyzed in this study were identified through an electronic search of the Cochrane library and the PubMed, Wanfang, China Biology Medicine (CBM), and Chinese National Knowledge Infrastructure (CNKI) databases. The search terms were "multiple myeloma", "thalidomide and VAD", "chemotherapy", "thalidomide", and "vascular endothelial growth factor". There were no language and date restrictions in the selection of studies. The initial search was performed from January 2000 to July 2017. Our search was based on Preferred Reporting Items for Systematic Reviews and Meta-Analyses (PRISMA) guidelines [20].

The selection criteria for this study were as follows: (1) randomized or nonrandomized controlled trials (RCTs or NRCTs) of patients with multiple myeloma and (2) trials in which patients in the experimental group received the $\mathrm{T}$ VAD regimen while patients in one control group were treated using VAD alone.

2.2. Data Extraction and Quality Assessment. Data were extracted independently by two reviewers, and any disagreements were discussed with a third investigator. The following data were collected: the first author's name, year of publication, clinical stage, chemotherapy regimens, number of subjects, patient age, test conditions, and treatment regimen units. Quality of the RCTs was critically appraised using the Cochrane Collaboration tool for assessing risk of bias (RoB) [21]. Studies were assessed based on the Cochrane Handbook by recording bias risks associated with 6 protocol components: random sequence generation, allocation concealment, blinding of participants, blinding of outcome assessment, incomplete outcome data, and selective reporting. Each of the six items was scored as "low risk", "unclear risk", or "high risk" [22]. The NRCTs were appraised using a modified RoB form adjusted to fit the nonrandomized study design. A three-point scale together with summarizing arguments for grading (low RoB, high RoB, or unclear) were used to assess each domain.
2.3. Curative Effect Evaluation. Primary outcome was serum VEGF level of nontumor populations, MM patients before treatment, and MM patients treated by VAD or T-VAD.

2.4. Statistical Analysis. Data were analyzed using Review Manager Version 5.2 provided by the Cochrane Collaboration. A $\mathrm{P}<0.05$ was considered statistically significant. Heterogeneity among the studies was assessed to determine the most suitable model [23]. When heterogeneity existed, a random-effects method was used; otherwise, a fixed-effects method was used. To evaluate whether the results of the studies were homogenous, we performed Inverse Variance in which homogeneity was considered present at $\mathrm{I}^{2}<50 \%$ or $\mathrm{P}>0.1$. Weighted mean difference (WMD) was the principal measure of effect and is presented with a 95\% confidence interval (CI).

\section{Results}

3.1. Search Results. A total of 2370 articles were identified during the initial search. After title and abstract review, 2332 articles were excluded because they were not clinical trials $(n=1023)$, were duplicates $(n=451)$, or did not measure the primary outcome $(n=858)$. In total, 38 studies were selected as potentially relevant. After full-text review, 25 articles were eliminated for insufficient data. Finally, 13 trials with a total of 815 patients were judged eligible for inclusion in this metaanalysis. The reasons for exclusion are illustrated in Figure 1.

3.2. Patient Characteristics. The 13 controlled trials included a total of 815 patients [24-36]. Five controlled trials (one randomized and four nonrandomized) including 293 patients were pooled, and data of the $179 \mathrm{MM}$ patients before treatment were compared to the 114 nontumor controls (Table 1). Then, 8 controlled trials ( 6 randomized, 1 nonrandomized, and 1 historical trial) including $481 \mathrm{MM}$ patients were pooled, and data of 263 patients treated with the T-VAD regimen were compared to 245 patients treated with the VAD regimen (Table 2; 29 patients from one randomized and one nonrandomized trial included in the first comparison of Table 1 were also included in the second comparison of Table 2). Finally, data from 2 randomized controlled trials including 70 VADT-treated patients were pooled, and data of 56 patients with initial MM were compared to 14 patients with recurrent MM (Table 3).

3.3. Quality Assessment. The risk of bias assessment is shown in Figures 2(a) and 2(b). Overall risk of bias was judged to be low or unclear in the included RCTs. Some of the included studies were described as open-label, or blinding was not reported at all. None of the RCTs fulfilled all six criteria for low RoB; that is, no study described randomization procedures, allocation concealment, or selective reporting with a low RoB (Figure 2(a)). For NRCT and HCTs, the research design was deemed suitable, with low RoB (Figure 2(b)).

3.4. Efficacy Assessments. The forest plot analyses of these comparisons are shown in Figures 1-6. Serum VEGF was significantly higher in MM patients than nontumor controls 


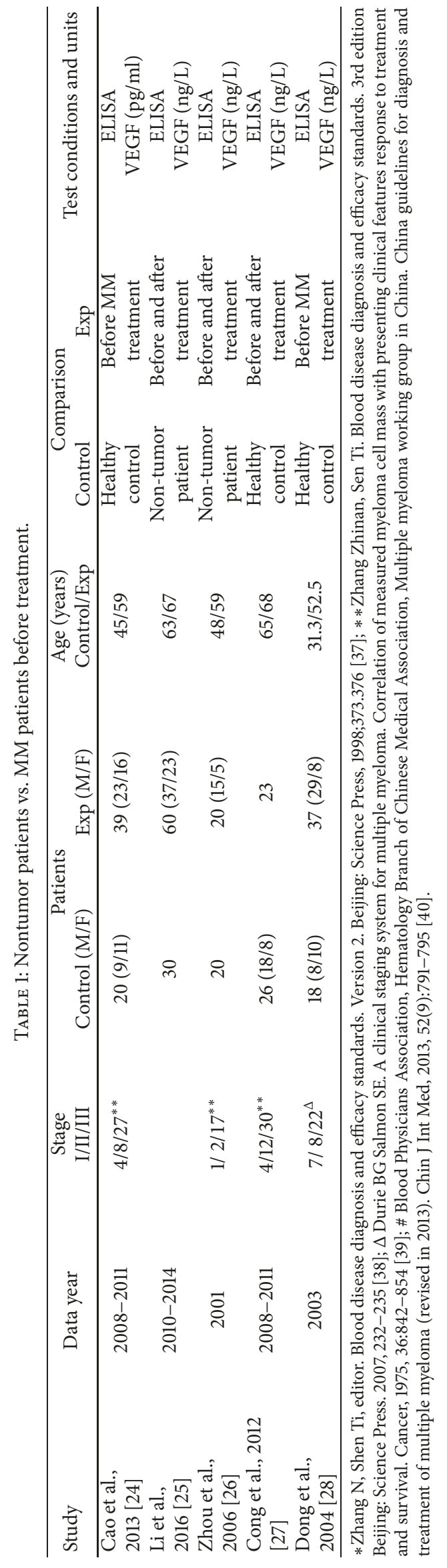


TABLE 2: VAD-treated vs. T-VAD-treated MM patients.

\begin{tabular}{|c|c|c|c|c|c|c|c|}
\hline \multirow{2}{*}{ Study } & \multirow{2}{*}{$\begin{array}{l}\text { Stage } \\
\text { I/II/III }\end{array}$} & \multicolumn{2}{|c|}{ Patients } & \multirow{2}{*}{$\begin{array}{l}\text { Age (years) } \\
\text { Control/Exp }\end{array}$} & \multicolumn{2}{|c|}{ Comparison } & \multirow{2}{*}{$\begin{array}{c}\text { Trial conditions and } \\
\text { units }\end{array}$} \\
\hline & & Control (M/F) & $\operatorname{Exp}(\mathrm{M} / \mathrm{F})$ & & Control & Exp & \\
\hline Cong et al., 2012 [27] & $4 / 12 / 30^{* *}$ & 23 & 23 & $65 / 68$ & VAD & T-VAD & $\begin{array}{c}\text { ELISA } \\
\text { VEGF (ng/L) }\end{array}$ \\
\hline Yang et al., 2015 [29] & $6 / 26 / 58^{* *}$ & $43(23 / 20)$ & $47(25 / 22)$ & $67.4 / 66.9$ & VAD & T-VAD & $\begin{array}{c}\text { ELISA } \\
\text { VEGF }(\mathrm{pg} / \mathrm{ml})\end{array}$ \\
\hline Long et al., 2015 [30] & $0 / 19 / 71^{*}$ & $45(26 / 19)$ & $45(27 / 18)$ & $56.4 / 55.3$ & VAD & T-VAD & $\begin{array}{c}\text { ELISA } \\
\text { VEGF (ng/L) }\end{array}$ \\
\hline Yuan et al., 2004 [31] & $10 / 31 / 12^{*}$ & $30(18 / 12)$ & $23(15 / 8)$ & $62 / 61$ & VAD & T-VAD & $\begin{array}{c}\text { ELISA } \\
\text { VEGF (ng/L) }\end{array}$ \\
\hline Liu et al., 2016 [32] & $0 / 24 / 26^{*}$ & $25(12 / 13)$ & $25(13 / 12)$ & $67.1 / 67.1$ & VAD & T-VAD & $\begin{array}{c}\text { ELISA } \\
\text { VEGF }(\mathrm{pg} / \mathrm{ml})\end{array}$ \\
\hline Tan et al., 2008 [33] & $11 / 33 / 12^{*}$ & $28(16 / 12)$ & $28(15 / 13)$ & $62 / 61$ & VAD & T-VAD & $\begin{array}{c}\text { ELISA } \\
\text { VEGF (ng/L) }\end{array}$ \\
\hline Yang et al., 2005 [34] & $0 / 8 / 76^{\Delta}$ & $45(31 / 14)$ & $39(29 / 10)$ & $51.5 / 52.3$ & VAD & T-VAD & $\begin{array}{c}\text { ELISA } \\
\text { VEGF (ng/L) }\end{array}$ \\
\hline Dong, 2004 [28] & $7 / 8 / 19^{\#}$ & 6 & 6 & $31.3 / 49.8$ & VAD & T-VAD & $\begin{array}{c}\text { ELISA } \\
\text { VEGF (ng/L) }\end{array}$ \\
\hline
\end{tabular}

TABLE 3: Initial MM vs. recurrent MM patients treated with T-VAD.

\begin{tabular}{|c|c|c|c|c|c|c|c|c|c|}
\hline \multirow{2}{*}{ Study } & \multirow{2}{*}{ Data year } & \multirow{2}{*}{$\begin{array}{c}\text { Tumor stage } \\
\text { I/II/III }\end{array}$} & \multicolumn{2}{|c|}{ Patients } & \multicolumn{2}{|c|}{ Age (year) } & \multicolumn{2}{|c|}{ Comparison } & \multirow{2}{*}{$\begin{array}{l}\text { Test conditions } \\
\text { and units }\end{array}$} \\
\hline & & & Initial & Relapse & Initial & Relapse & Initial & Relapse & \\
\hline $\begin{array}{l}\text { Zhai et al., } 2012 \\
\text { [35] }\end{array}$ & 2004-2011 & $0 / 13 / 29^{*}$ & 30 & 7 & 56 & 56 & T-VAD & T-VAD & $\begin{array}{c}\text { ELISA } \\
\text { VEGF }(\mathrm{pg} / \mathrm{ml})\end{array}$ \\
\hline $\begin{array}{l}\text { Du et al., } \\
2014 \text { [36] }\end{array}$ & Not clear & $0 / 6 / 32^{\#}$ & 26 & 7 & 58 & 58 & T-VAD & T-VAD & VEGF $(\mathrm{pg} / \mathrm{ml})$ \\
\hline
\end{tabular}

*Zhang N, Shen Ti, editor. Blood disease diagnosis and efficacy standards. Version 2. Beijing: Science Press, 1998;373.376 [37]; **Zhang Zhinan, Sen Ti. Blood disease diagnosis and efficacy standards. 3rd edition Beijing: Science Press. 2007, 232-235 [38]; $\Delta$ Durie BG Salmon SE. A clinical staging system for multiple myeloma. Correlation of measured myeloma cell mass with presenting clinical features response to treatment and survival. Cancer, 1975, 36:842-854 [39]; \# Blood Physicians Association, Hematology Branch of Chinese Medical Association, Multiple myeloma working group in China. China guidelines for diagnosis and treatment of multiple myeloma (revised in 2013). Chin J Int Med, 2013, 52(9):791-795 [40].

$(\mathrm{MD}=353.01,[187.52-518.51], \mathrm{P}<0.01)$ (Figure 3). Overall efficacy of the T-VAD regimen was higher than that of the $\mathrm{VAD}$ regimen $(\mathrm{RR}=1.36,[1.21-1.53], \mathrm{P}<0.01)$ (Figure 4). Further, the T-VAD regimen was more effective for decreasing serum VEGF than the VAD regimen $(\mathrm{MD}=-49.85,[-66.28-$ -33.42], $\mathrm{P}<0.01$ ) (Figure 5). The T-VAD regimen was also more effective for reducing serum VEGF in initial MM than recurrent $\mathrm{MM}$, even when treatment was curative in both groups $(\mathrm{MD}=-120.20,[-164.60--39.80], \mathrm{P}<0.01)$ (Figure 6). Alternatively, there was no significant difference in marrow microvessel density (MVD) reduction between initial MM and recurrent MM patient groups, both showing curative results $(\mathrm{MD}=175.94,[-26.08-377.95], \mathrm{P}>0.01)$ (Figure 7). Finally, two courses of T-VAD reduced serum VEGF to the level of a nontumor control group ( $\mathrm{MD}=175.94$, [26.08-377.95], $\mathrm{P}=0.09$ ) (Figure 8).

\section{Discussion}

Vascular endothelial growth factor is a potent inducer of bone marrow angiogenesis, suggesting a significant role in $\mathrm{MM}$ pathogenesis. Indeed, high serum VEGF is an independent risk factor for poor MM prognosis, while reduced VEGF is related to the curative effect of treatment [4]. However, there is no large-sample evidence that the antiangiogenic $\mathrm{T}$ $\mathrm{VAD}$ regimen is superior to the conventional VAD regimen for reducing serum VEGF or if the difference in VEGF suppression is related to the difference in antitumor efficacy. Therefore, we conducted this meta-analysis of 13 controlled trials. Results indicated that serum VEGF is higher in MM patients than nontumor controls (Figure 3). This suggests that myeloma cells release more VEGF, consistent with previous findings that overexpression of VEGF and aberrant angiogenesis in bone marrow are closely related to MM pathogenesis [41-43]. Thus, new targeted therapies that treat MM by adjusting the bone marrow microenvironment to suppress angiogenesis may be advantageous compared to traditional chemotherapy regimens. Thalidomide was added to the VAD regimen based on its efficacy for antiangiogenesis, for immunosuppression, and for adjusting the bone marrow microenvironment $[6,44,45]$, and clinical studies have shown that the overall efficacy of T-VAD for MM is up to $70 \%[7,46]$. This regimen is also relatively inexpensive and so is particularly valuable for MM treatment in developing countries. This meta-analysis shows that the curative efficacy of T-VAD (76.92\% to $88.9 \%$ ) is greater than VAD (Figure 4 ) 


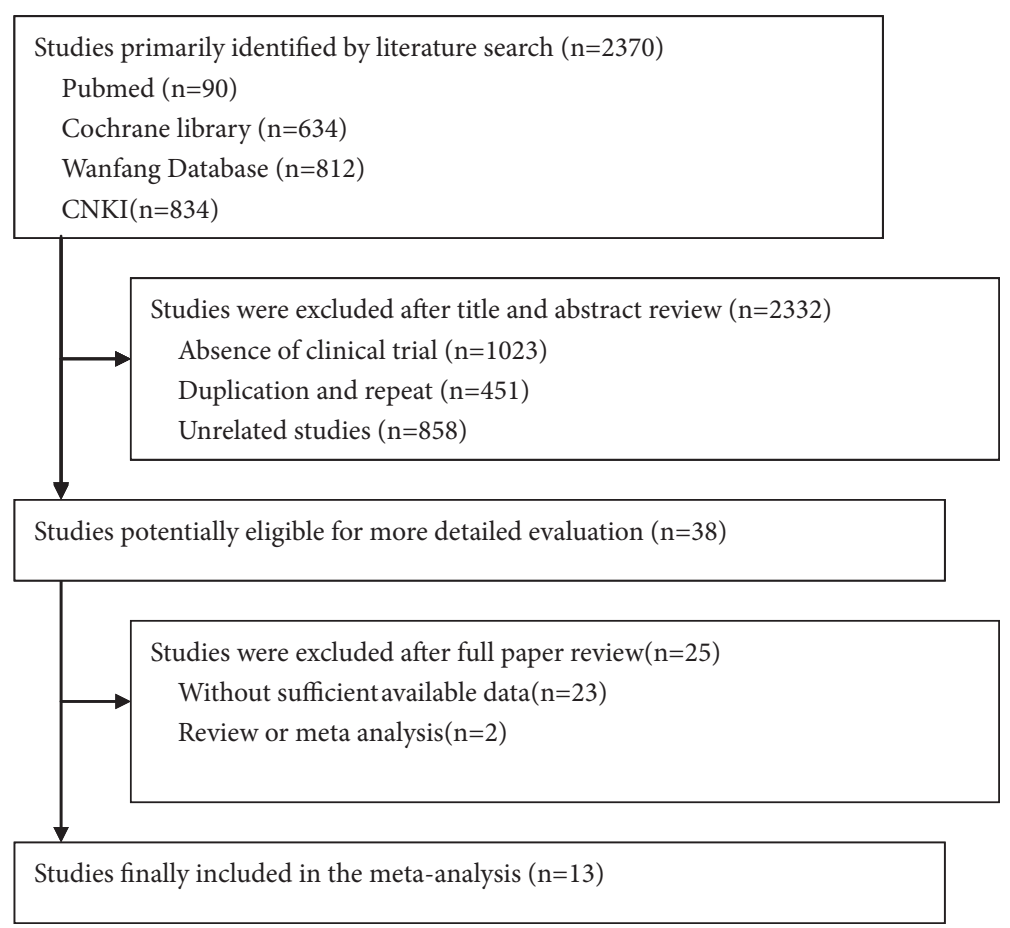

FIGURE 1: Preferred Reporting Items for Systematic Reviews and Meta-Analyses (PRISMA) flow diagram.
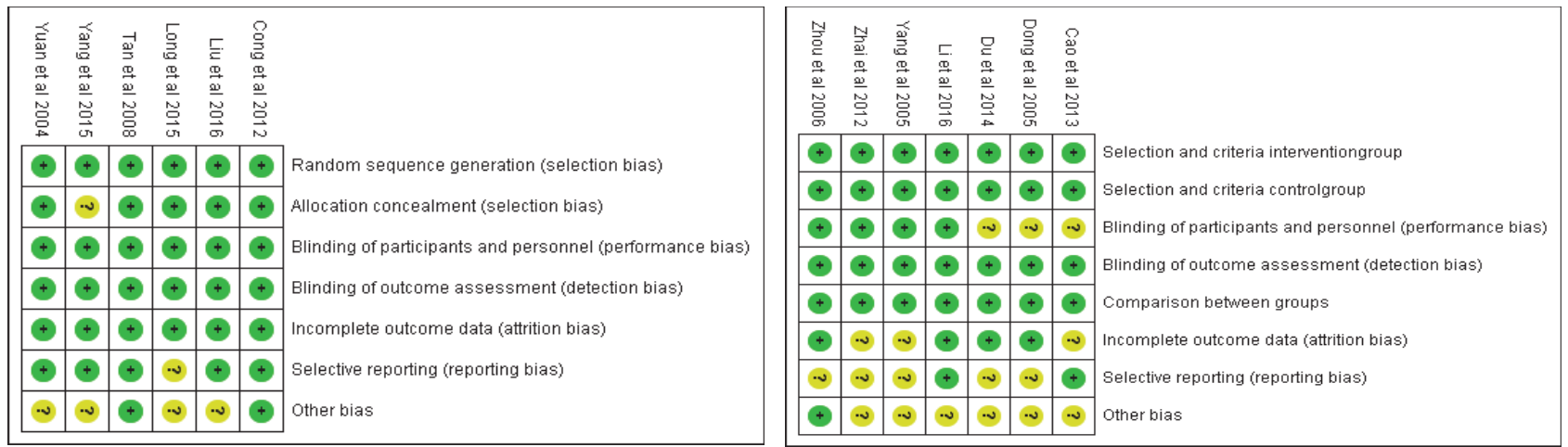

FIGURE 2: The assessment of the risk of bias.

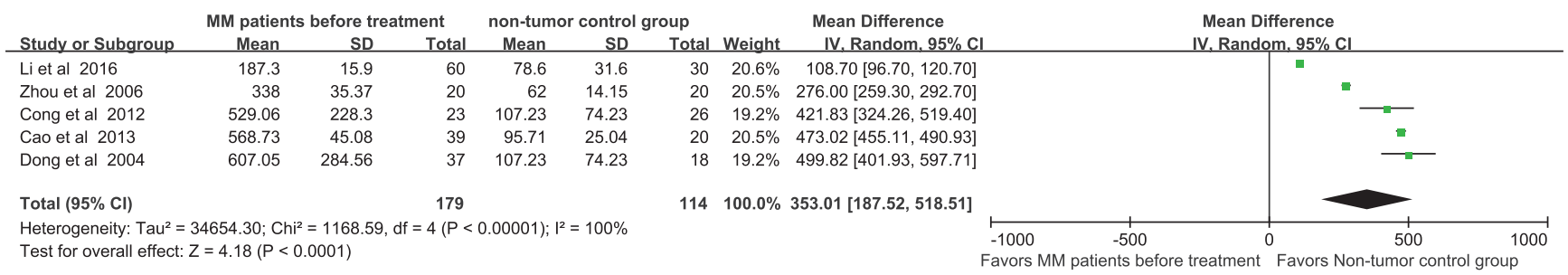

FIgURE 3: Forest plot comparing serum VEGF between MM patients before treatment and a nontumor control group.

and also more effective than VAD for reducing serum VEGF (Figure 5), suggesting that the enhanced curative efficacy is linked to reduction in serum VEGF.

This meta-analysis also addressed whether the efficacy of T-VAD for reducing serum VEGF is the same for patients with initial and recurrent MM. The decrease in serum VEGF was significantly greater during initial MM than recurrent MM despite curative effects in both groups (Figure 6). Alternatively, there was no difference in MVD reduction (Figure 7) between initial and current MM patients effectively treated with T-VAD. Thus, serum VEGF reduction appears to be a more reliable index of treatment efficacy for initial 


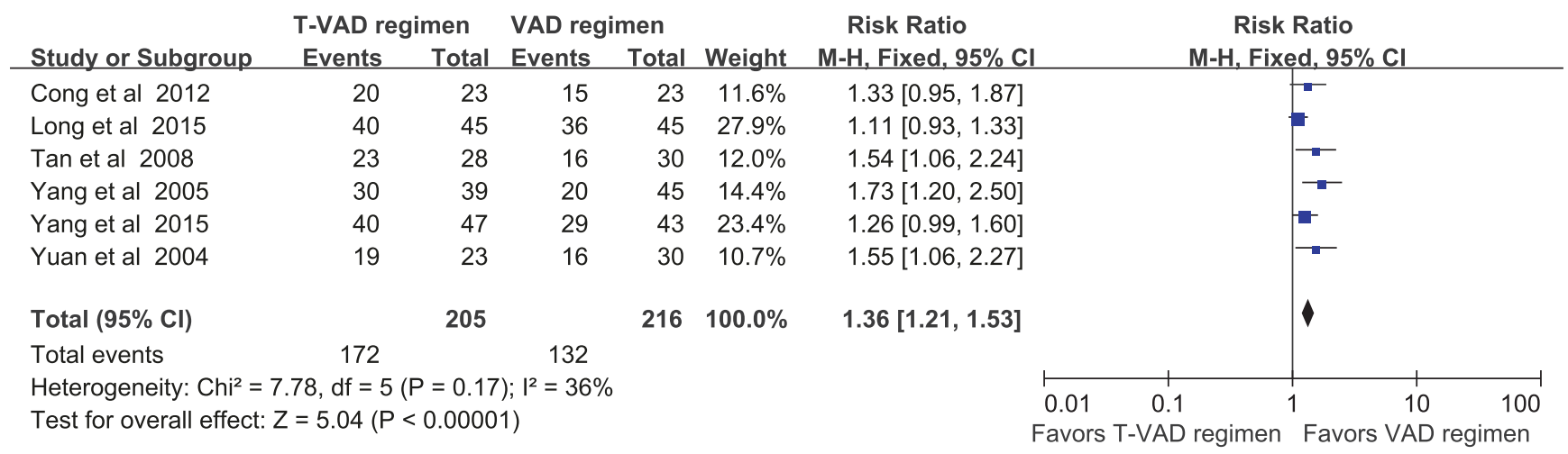

FIGURE 4: Forest plot comparing overall efficacy of the T-VAD regimen to the VAD regimen for MM treatment.

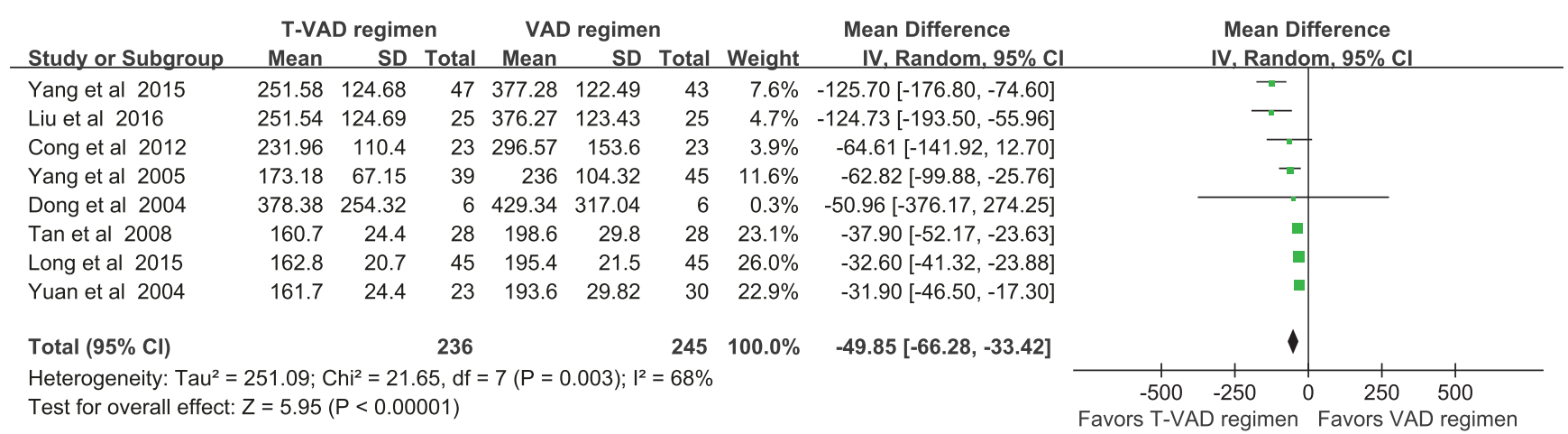

FIGURE 5: Forest plot comparing serum VEGF levels in MM patients treated with the T-VAD regimen or the VAD regimen.

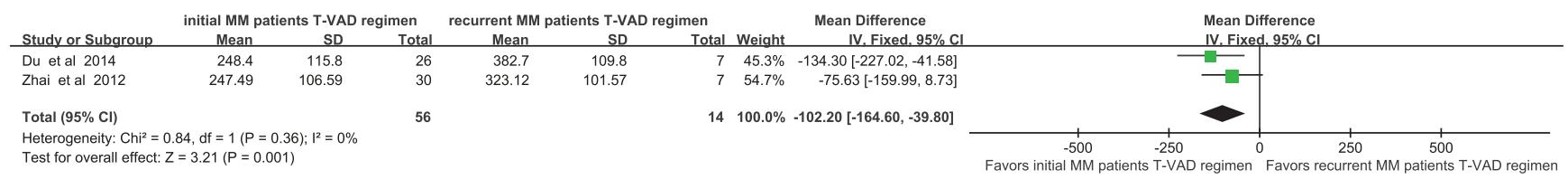

FIGURE 6: Forest plot comparing serum VEGF between initial and recurrent MM patients treated effectively with the T-VAD regimen [defined as complete remission (CR), very good partial remission (VGPR), or partial remission].

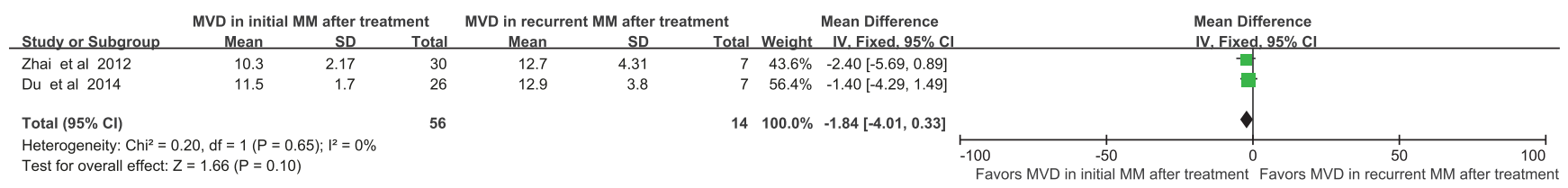

FIGURE 7: Forest plot comparing marrow microvessel density (MVD) between initial and recurrent MM patients both treated effectively with the T-VAD regimen.

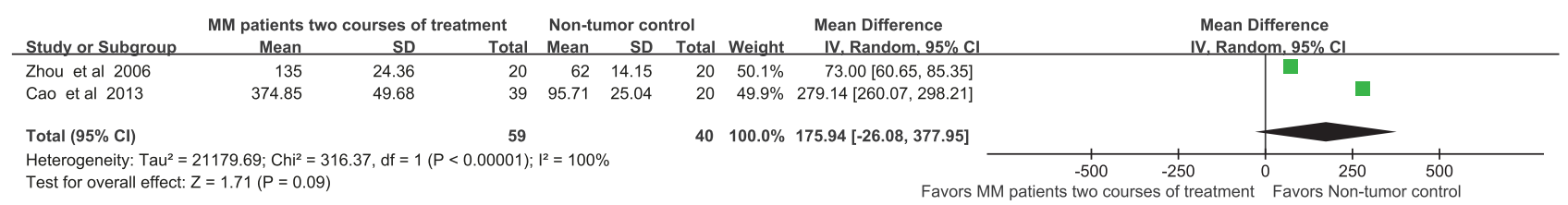

FIGURE 8: Forest plot comparing serum VEGF levels between MM patients after two courses of T-VAD treatment and nontumor controls. 
MM than recurrent MM, although antiangiogenesis appears important for good clinical outcome in both cases. The analysis conducted by the point that the VEGF expression level in bone marrow of multiple myeloma patients is consistent with MVD shows that it is consistent with the viewpoint of referencing it in the treatment of relapsed/refractory MM without standard treatment strategy [47].

Finally, there was no significant difference between serum VEGF in MM patients treated with two courses of T-VAD and healthy controls, underscoring the efficacy of T-VAD for reducing serum VEGF and further implicating elevated serum VEGF in MM pathogenesis and prognosis. However, the $\mathrm{P}$ value is relatively small, which may be caused by insufficient sample size, and the possibility that some malignant myeloma cells still remain in the body after effective treatment cannot be excluded. These residual myeloma cells will still release VEGF, and residual VEGF may be one factor promoting recurrence, an issue that requires further study [27].

This meta-analysis has certain limitations. Most of the trials included in the study were conducted in China, which may be related to the low price of this regimen. Additionally, MM mainly afflicts the elderly, so incidence may be related to population aging in China [8]. Moreover, the sample size for the efficacy indicator (serum VEGF) may not be sufficient to detect smaller differences among groups (Figures 4-6). Finally, this analysis did not consider toxic side effects $[8,9]$. Given that the side effects of the VAD regimen are significant, it is necessary to develop alternatives.

\section{Conclusion}

This meta-analysis indicates that serum VEGF is a clinically significant factor influencing the occurrence, development, and prognosis of multiple myeloma and that the antiangiogenic T-VAD regimen is a better MM treatment than VAD alone. This superior efficacy is associated with greater suppression of serum VEGF. Thus, serum VEGF is an important indicator of treatment efficacy for first-time MM. Larger scale studies are still required to confirm the superior efficacy of T-VAD and the clinical significance of serum VEGF for MM prognosis and treatment outcome.

\section{Data Availability}

The data supporting this meta-analysis are from previously reported studies, which have been cited. The processed data are available in the forest plot.

\section{Conflicts of Interest}

The authors declare that they have no conflicts of interest.

\section{Authors' Contributions}

Juan Li conceived and designed the experiments; Gan-Lin He and Sui-Zhi He analyzed the data; Duo-Rong Xu and Wai-Yi Zou collected the data; and Gan-Lin He wrote the paper.

\section{References}

[1] J. L. Harousseau and M. Attal, "How i treat first relapse of myeloma," Blood, vol. 130, no. 8, pp. 963-973, 2017.

[2] R. A. Kyle and S. V. Rajkumar, "Multiple myeloma," Blood, vol. 111, no. 6, pp. 2962-2972, 2008.

[3] P. Moreau, "How I treat myeloma with new agents," Blood, vol. 130, no. 13, pp. 1507-1513, 2017.

[4] P. Moreau, J. San Miguel, P. Sonneveld et al., "Multiple myeloma: ESMO Clinical Practice Guidelines for diagnosis, treatment and follow-up," Annals of Oncology, vol. 28, pp. iv52-iv61, 2017.

[5] M. M. McDonald, M. R. Reagan, S. E. Youlten et al., "Inhibiting the osteocyte-specific protein sclerostin increases bone mass and fracture resistance in multiple myeloma," Blood, vol. 129, no. 26, pp. 3452-3464, 2017.

[6] N. Becker, "Epidemiology of multiple myeloma," Recent Results in Cancer Research, vol. 183, pp. 25-35, 2011.

[7] Y. Zhao, L.-P. Dou, S.-H. Wang et al., "The efficacy and safety of PAD and VAD regimens for untreated multiple myeloma," Zhonghua nei ke za zhi [Chinese journal of internal medicine], vol. 49, no. 9, pp. 762-764, 2010.

[8] X. J. Huang, "Current status and challenges of treatment of multiple myeloma in China," in Chinese Journal of Hematology, vol. 34, pp. 281-282, 2013.

[9] S. W. Lane, D. Gill, P. N. Mollee, and S. V. Rajkumar, "Role of VAD in the initial treatment of multiple myeloma (multiple letters)," Blood, vol. 106, no. 10, pp. 3674-3675, 2005.

[10] A. Vacca, D. Ribatti, L. Roncali et al., "Bone marrow angiogenesis and progression in multiple myeloma," British Journal of Haematology, vol. 87, no. 3, pp. 503-508, 1994.

[11] J.-R. Liu, S.-K. Luo, J. Li, and C. Su, "Expression and clinical significance of vascular endothelial growth factor and its receptors in multiple myeloma," Ai zheng = Aizheng = Chinese journal of cancer, vol. 26, no. 6, pp. 652-656, 2007.

[12] N. F. Andersen, U. Vogel, T. W. Klausen et al., "Vascular endothelial growth factor (VEGF) gene polymorphisms may influence the efficacy of thalidomide in multiple myeloma," International Journal of Cancer, vol. 131, no. 5, pp. E636-E642, 2012.

[13] R. Seki, S.-I. Yamagishi, T. Matsui et al., "Pigment epitheliumderived factor (PEDF) inhibits survival and proliferation of VEGF-exposed multiple myeloma cells through its antioxidative properties," Biochemical and Biophysical Research Communications, vol. 431, no. 4, pp. 693-697, 2013.

[14] X. Li, X.-Z. Wei, J.-W. Liu, C.-Y. Geng, and W.-M. Chen, "[Clinical significance of serum vascular endothelial growth factor in patients with multiple myeloma]." Zhongguo shi yan xue ye xue za zhi / Zhongguo bing li sheng li xue hui= Journal of experimental hematology / Chinese Association of Pathophysiology, vol. 22, no. 1, pp. 108-111, 2014.

[15] N. Mimura, T. Hideshima, and K. C. Anderson, "Novel therapeutic strategies for multiple myeloma," Experimental Hematology, vol. 43, no. 8, pp. 732-741, 2015.

[16] Y. Zhuang and Q. Shen, "Latest advances on the maintenance therapy of multiple myeloma," Zhongguo shi yan xue ye xue za zhi / Zhongguo bing li sheng li xue hui = Journal of experimental hematology / Chinese Association of Pathophysiology, vol. 23, no. 1, pp. 250-254, 2015.

[17] S. Giralt, "A TAD better for myeloma therapy?” Blood, vol. 115, no. 6, pp. 1109-1110, 2010. 
[18] W. Wang and H.-B. Liu, "Efficacy Comparison of Low dose Thalidomide Combined with Modified VCMP and VAD regimens for Treatment of Aged MM Patients," Zhongguo shi yan xue ye xue za zhi / Zhongguo bing li sheng li xue hui= Journal of experimental hematology / Chinese Association of Pathophysiology, vol. 24, no. 3, pp. 765-768, 2016.

[19] A. Maiolino, V. T. M. Hungria, M. Garnica et al., "Thalidomide plus dexamethasone as a maintenance therapy after autologous hematopoietic stem cell transplantation improves progressionfree survival in multiple myeloma," American Journal of Hematology, vol. 87, no. 10, pp. 948-952, 2012.

[20] D. Moher, A. Liberati, J. Tetzlaff, and D. G. Altman, "Preferred reporting items for systematic reviews and meta-analyses: the PRISMA statement," PLoS Medicine, vol. 6, no. 7, Article ID e1000097, 2009.

[21] "Cochrane Handbook for Systematic Reviews of Interventions Ver-sion 5. 1. 0," March 2011, http://www.cochranehandbook.org.

[22] X. Zeng, Y. Zhang, J. S. Kwong et al., "The methodological quality assessment tools for preclinical and clinical studies, systematic review and meta-analysis, and clinical practice guideline: a systematic review," Journal of Evidence-Based Medicine, vol. 8, no. 1, pp. 2-10, 2015.

[23] D. Jackson, I. R. White, and R. D. Riley, "Quantifying the impact of between-study heterogeneity in multivariate meta-analyses," Statistics in Medicine, vol. 31, no. 29, pp. 3805-3820, 2012.

[24] LX. Cao and Z. Xiao, "Observe the efficacy of thalidomide combined with VAD Programtreat on multiple myeloma patient and in the impact of IL-6 and VEGF," Journal of Logistics University of CAPF (Medical Sciences), vol. 22, no. 7, pp. 617-622, 2013.

[25] Z. Z. Li, H. X. Chai, and Y. Y. He, "The expression of Survivin and VEGF in multiple myeloma and their relationship," Journal of Modern Oncology, vol. 24, no. 20, pp. 3281-3284, 2016.

[26] Y. X. Zhou, Clinical Study on Thalidomide in the treatment of Multiple Myeloma [Master Thesis], Qingdao University, 2006.

[27] L. Cong, J. Xiao, W.-J. Zhang et al., "Expressions and clinical significance of VEGF, TNF- $\alpha$ and IL- 6 in MM patients with thalidomide and VAD chemotherapy," Chinese Journal of Cancer Prevention and Treatment, vol. 19, no. 5, pp. 375-377, 2012.

[28] L. H. Dong, Clinical significance of serum vascular endothelial growth factor and interleukin-6 in MM [Master, thesis], Central South University, pp. 6-11, 2004.

[29] Z. F. Yang, Y. Li, X. Y. Liu et al., Influence of low dose thalidomide maintenance treatment on curative effect and serum related indexes in multiple myeloma, vol. 24, China Pharmaceuticals, 2015.

[30] B. B. Long, "Effect of thalidomide combined with VAD regimen on multiple myeloma Security analysis," Contemporary Medicine, vol. 21, pp. 127-128, 2015.

[31] Y. N. Yuan and Q. W. Zhu, "The clinical research of thalidomide incorporation with VAD in treatment of multiple myeloma," Journal of Clinical Hematology, vol. 17, no. 4, pp. 209-211, 2004.

[32] J. Y. Liu, C. P. Wang, C. L. Wang et al., "A small dose of thalidomide and VAD therapy for multiple myeloma," International Medicine and Health Guidance News, vol. 22, pp. 2820-2822, 2016.

[33] Q. Wang, X. X. Zhu, and C. B. Zhang, "Experimental study on honeysuckle extract against bacteria," Chinese Journal of Medical Guide, vol. 10, no. 9, pp. 1428-1430, 2008.

[34] Y. J. Yang, Clinical Analysis of Thalidomide in Combination with VAD to Treat Refractory or Relapsed Multiple Myeloma. Jilin
University [Master thesis], Jilin University, Changchun, China, 2005.

[35] X. H. Zhai, Y. Wang, X. H. Lian et al., "Clinical observation of low-dose thalidomide in the treatment of multiple myeloma and its effect on VEGF and MVD," Shanxi Medical Journal, vol. 41, no. 2, pp. 216-218, 2012.

[36] Z. Y. Du and R. Y. Yang, "Clinical observation of thalidomide maintenance therapy for multiple myeloma," Chinese Journal of Practical Medicine, vol. 41, pp. 54-56, 2014.

[37] N. Zhang and T. Shen, Blood disease diagnosis and efficacy standards. Version 2, Science Press, Beijing, China, 1998.

[38] Z. N. Zhang and T. Sen, Blood disease diagnosis and efficacy standards, Science Press, Beijing, China, 3rd edition edition, 2007.

[39] B. G. M. Durie and S. E. Salmon, "A clinical staging system for multiple myeloma," Cancer, vol. 36, no. 3, pp. 842-854, 1975.

[40] Blood Physicians Association, Hematology Branch of Chinese Medical Association, and Multiple myeloma working group in China, "China guidelines of diagnosis and treatment of multiple myeloma (revised in 2013)," Chinese Journal of Internal Medicine, vol. 52, no. 9, pp. 791-795, 2013.

[41] D. Marisavljevic, O. Markovic, V. Cemerikic, and D. Babic, "Clinical and histopathological study of angiogenesis in multiple myeloma," Journal of B.U.ON., vol. 16, no. 1, pp. 98-103, 2011.

[42] K. S. Cohen, "Angiogenesis and multiple myeloma: Is there a role for angiogenic biomarkers in the context of autologous stem cell transplant?” Leukemia \& Lymphoma, vol. 52, no. 7, pp. 1173-1175, 2011.

[43] C. Rana, S. Sharma, V. Agrawal, and U. Singh, "Bone marrow angiogenesis in multiple myeloma and its correlation with clinicopathological factors," Annals of Hematology, vol. 89, no. 8, pp. 789-794, 2010.

[44] A. Mercurio, G. Adriani, A. Catalano et al., "A mini-review on thalidomide: Chemistry, mechanisms of action, therapeutic potential and anti-angiogenic properties in multiple myeloma," Current Medicinal Chemistry, vol. 24, no. 25, pp. 2736-2744, 2017.

[45] M. A. Dimopoulos, A. Oriol, H. Nahi et al., "Daratumumab, lenalidomide, and dexamethasone for multiple myeloma," The New England Journal of Medicine, vol. 375, no. 14, pp. 1319-1331, 2016.

[46] G.-J. Li, H. Ding, and L. Yang, "Correlation of WT1 and VEGF Expression with Angiogenesis in Bone Marrow Biopsies of Multiple Myeloma Patients," Zhongguo shi yan xue ye xue za zhi / Zhongguo bing li sheng li xue hui = Journal of experimental hematology / Chinese Association of Pathophysiology, vol. 23, no. 3, pp. 733-736, 2015.

[47] W. Chen, "The guidelines for the diagnosis and management of multiple myeloma in China (2015 revision) : interpretation of the treatment of relapsing and refractory multiple myeloma," Zhonghua Nei Ke Za Zhi, vol. 55, no. 2, pp. 93-94, 2016. 


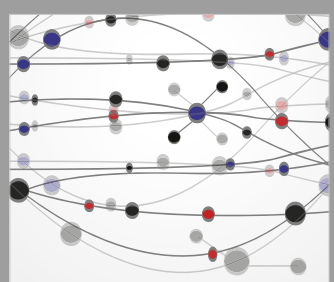

The Scientific World Journal
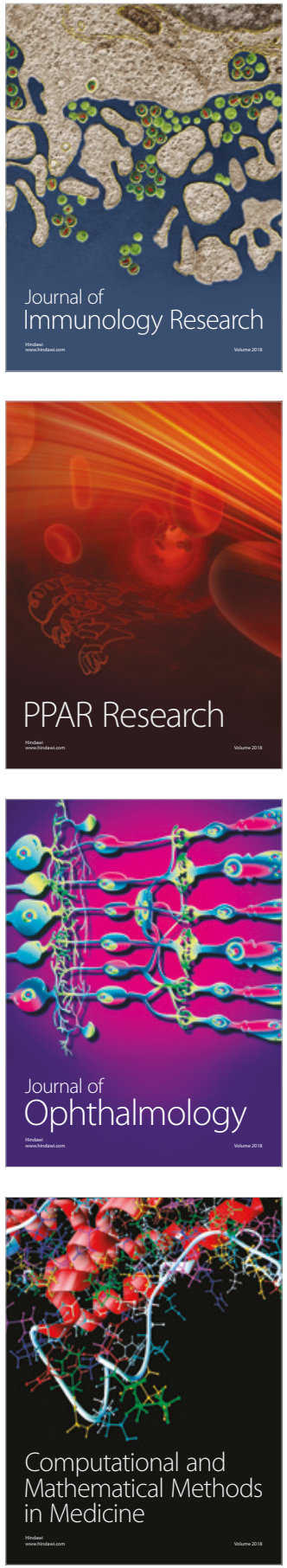

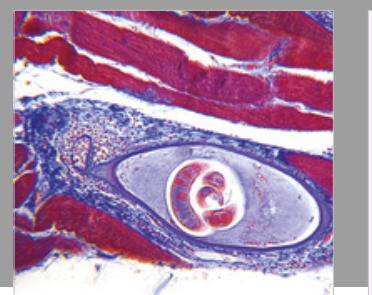

Gastroenterology Research and Practice

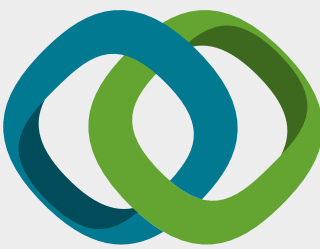

\section{Hindawi}

Submit your manuscripts at

www.hindawi.com
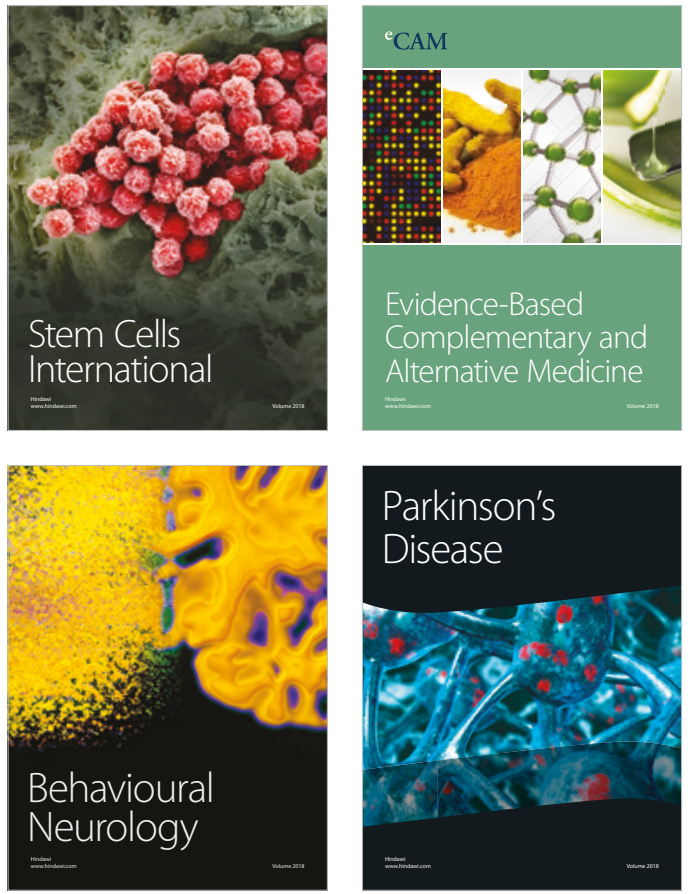

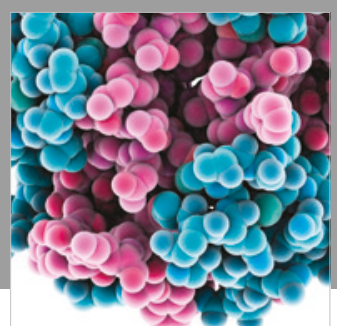

ournal of

Diabetes Research

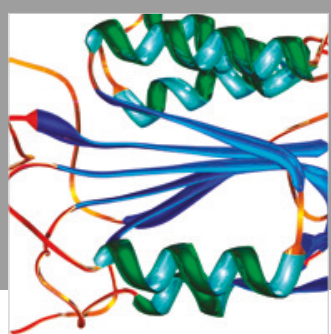

Disease Markers
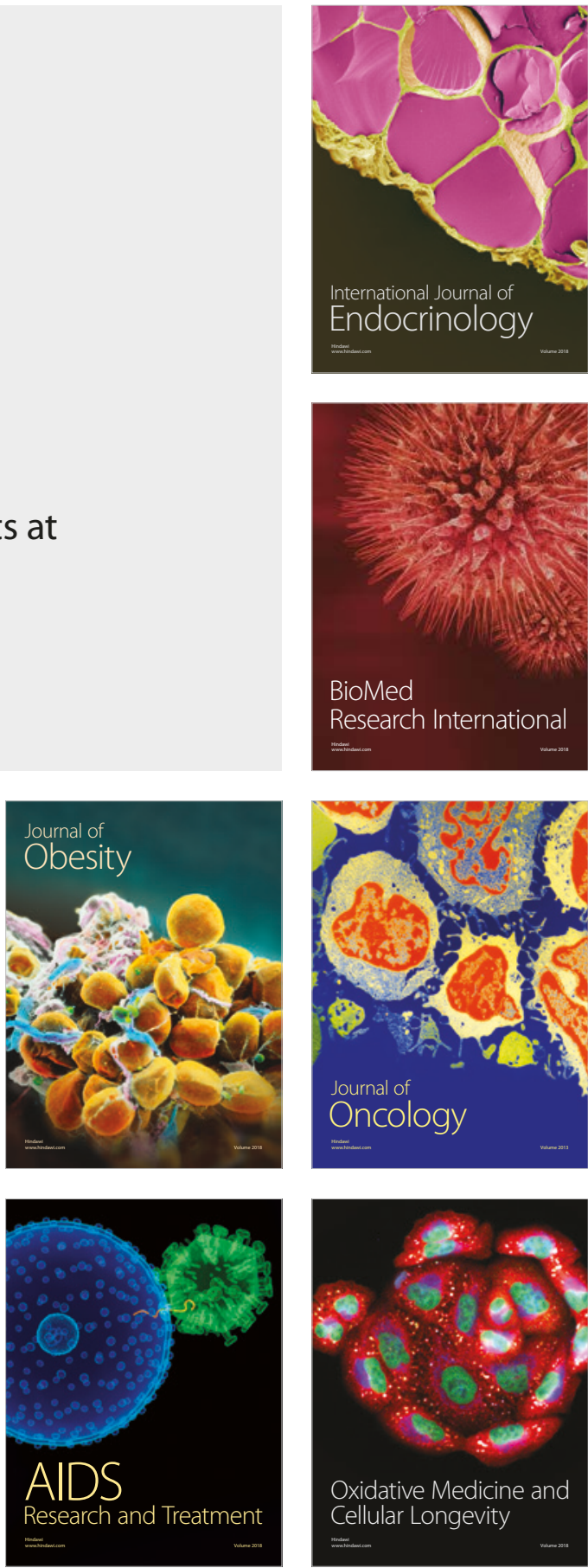